\title{
Efficiency Control Improvement Of Diesel Engines Conditions By Using The Method Of Analytical Synchronization Of Monitored Data
}

\author{
V Zalozh ${ }^{1}$, T Tarasenko ${ }^{1}$, R Varbanets ${ }^{2}$ \\ ${ }^{1}$ Danube institute of National university «Odessa maritime academy», Department of \\ Engineering Sciences, Fanagoriyskaya str. 9, Izmail, Ukraine, 68607 \\ zalogh@ukr.net zalozh86@gmail.com sergeysunnysat@gmail.com \\ ${ }^{2}$ Odessa National Maritime University, Department of Ship Power Plants and \\ Technical Operation, Mechnikova str. 34, Odessa, Ukraine, 65029 \\ roman.varbanets@gmail.com
}

\begin{abstract}
Improving the efficiency of the ship's diesel power plant is becoming increasingly important every year. This is primarily due to the restrictions on emissions of sulfur and nitrogen oxides into the atmosphere during the operation of the ship's diesel engines. Article is devoted to the problem of synchronization of data monitoring working process in transport diesel engines operation. The accuracy of data synchronization determines the error in determining power, control of the main engine systems operation and its diagnostics. The method of solving the synchronization problem must be analytical. The method of solving the synchronization problem must be analytical. In comparison the hardware methods used in most diagnostic systems of the working process of diesel engines have several disadvantages. It is impossible to conduct rapid diagnostic control in operation, non-stationary phase and amplitude errors in calculation the main parameters of the working process, the need for preparatory operations and related need for temporary withdrawal of diesel engine operation, complexity and high cost of diagnostic control. An analytical synchronization method is developed based on the top dead center coordinate determination algorithm, which uses three stages: linear, sinusoidal and model equal to zero the first derivative of the pressure under compression. The proposed method of analytical synchronization gives the obvious advantages for monitoring working process systems of diesel engines. The error in determining the main parameters of the working process and the indicator power does not exceed $2.5 \%$.

Keywords: marine diesel, effective power, top dead center, cylinder pressure monitoring and analyzing, mean indicated pressure, indicated power, analytical synchronization.
\end{abstract}

\section{Introduction}

Solving the problem of the analytical synchronization in view of monitoring the working process of transport diesel engines in operation is a great importance, because the operation of the main engine and its diagnosis is based on results of monitoring. The problem is formulated as the translation of data from time functions into functions by the angle of rotation of the crankshaft, where the accuracy of determining the top dead center (TDC) of the piston has the greatest influence $f(t) \stackrel{T D C}{\longrightarrow} f(\varphi)$ [2], [4]. 
There are two methods of solving this problem: hardware - using sensors on the engine flywheel (AVL OT-sensor 428 [10], Kistler TDC sensor Type 2629B [9], etc.) and analytical - by analyzing the curve $p(t)$ of the gas pressure in the working cylinder.

Hardware synchronization method is obvious and has been traditionally used to transport diesel from the beginning of using on them the first systems of monitoring the working process. The following system synchronized the data of monitoring using sensors on the wheels: one of the first widespread in the 1990 on the fleet of marine diesel monitoring systems NK-5 and subsequent NK100, NK-200 Norwegian company Autronica A/S; European systems Kyma, Premet, Malin, Doctor and many other systems developed before the early 2000s [6]. Then, when expensive portable stationary systems were replaced by portable periodic monitoring systems, analytical methods of synchronization began to develop.

It became apparent that the TDC hardware sensors, which are inconvenient when working with portable systems, also introduce a number of significant errors in the calculation of the average indicator pressure and indicator power. Installation and calibration of TDC sensors is carried out in statics. Then, when the engine is running under load, the TDC position recorded by the sensors is shifted due to torsional vibrations of the shaft and the selection of micro-backlash in the bearings of the crank mechanism. An additional, significant error is introduced by the pressure measuring channel - the indicator crane channel, which is installed on all low- and medium-speed diesel engines. All portable monitoring systems record the pressure in the working cylinder by attaching a sensor to the indicator valve, which has a standard thread on all transport diesels since receiving a patent by Rudolf Diesel in 1892. During pressure measurement, the channel introduces errors such as signal delay and throttling, and the first can be very significant - up to several degrees of crankshaft rotation (CA) - and increases with increasing speed [2]. Maintaining high economic efficiency and environmental safety in the operation of the diesel engine in modern conditions is becoming not only the norm but also a necessity. At the same time, effective, with minimal risk of accidents and damage to the environment, exploitation of marine diesel engine is inextricably linked to the diagnostic control of thermodynamic processes taking place inside it.

\section{Analyses of the Literature Data and the Problem Statement}

The error of the hardware sensors is so large and unstable depending on the load that it became obvious that within this error and even more accurately, the position of the TDC can be calculated by analyzing the curves $p(t)$. On the other hand, the influence of the TDC error on the accuracy of calculating the average indicator pressure is very large: $6-9 \%$ per $1^{\circ} \mathrm{TDC}$ error (M. Tazerout [12], S. Polanowski [14], Per Tunestal [15], Y. Nilsson and L. Eriksson [16], E. Pipitone [17]). Thus, in order to obtain an acceptable calculation of the average indicator pressure and indicator power, it is necessary to determine the TDC with a maximum absolute error of not more than $0,1-0,3^{\circ}$ degrees of crankshaft rotation[13].

The method proposed by the authors for solving the equation $P^{\prime}=0$, followed by the thermodynamic displacement of the TDC (M. Tazerout, E. Pipitone) [20], provides the required accuracy for calculating the TDC of $0,1-0,25^{\circ}$ degrees of crankshaft rotation . The curve $P^{\prime}=0$ is simulated from the closing of the intake valves to the start of ignition in the cylinder. Appropriate boundaries are determined by analyzing the extremes of the curves $\frac{d P}{d t}, \frac{d^{2} P}{d t^{2}}$, which is possible after filtering the initial pressure curve[5]. The authors apply digital filter Butterworth Low Pass filter that has a smooth characteristic across the frequency range and does not offset the phase of the original signal. This ensures the required accuracy of the final calculation of the average indicator pressure with the subsequent calculation of the indicator and effective engine power with a maximum relative error of no more than $2,5 \%$ [2].

Calculation of the effective power on marine diesel engines is necessary not only for assessing specific fuel consumption and diagnosing the technical condition, but also for the currently relevant calculation of the energy efficiency coefficient [27] of ships introduced by the IMO international 
maritime organization. IMO Resolution MEPC.282 (70) provides guidance on the development of a shipboard energy efficiency management plan (SEEMP - Ship Energy Efficiency Management Plan), which proposes approaches to managing environmental friendliness and efficiency, as well as possible ways to improve the overall performance of the vessel [7]. The main factors influencing to the energy efficiency, set out in a Resolution applicable for inland vessels in varying degrees of "weight". Fundamentally, all possible methods of monitoring energy efficiency in accordance with Annex VI of the MARPOL Convention, applied to ships in service, with certain assumptions fair for inland navigation vessels.

IMO activity and the actual volumes of $\mathrm{CO}_{2}$ emission reduction recognized by MEPC as a result of the implementation of measures to improve energy efficiency indicators are also relevant for inland vessels [28]. This task is gradually reflected in shipping development projects [4] on the conventional (Rhine, Danube, Sava, Moselle) inland waterways of Europe, primarily in environmental projects. Currently, over 550 million tons of cargo are transported annually on the inland waterways of the countries of the European Union .

Thus, the problem of analytical synchronization required for the correct assessment of power and technical diagnostics that improve the economic and environmental performance of transport diesel engines, is relevant [11].

\section{Aims}

The aim of this work is to present a method of analytical synchronization of data monitoring working process in transport diesel engines operation, based on an algorithm for determining the coordinate of the top dead centre, which unlike the existing ones, uses three stages: linear, sinusoidal and model model equal to zero the first derivative of the pressure under compression which provides the calculation of the indicator power and the main parameters of the working process with a maximum relative error of up to $2.5 \%$ is developed.

\section{Studying the Analytical Methods of the TDC Determining}

Analytical data synchronization method based on the fact that in the absence of fuel supply rate of change of pressure in the piston top dead center is equal to zero.

4.1 Linear Regression Method. The TDC method can be explained if we consider the diagram $P(\varphi, d e g)$ of a typical medium-speed marine engine and its derivative $d P / d \varphi$, recorded using highquality IMES pressure sensors [8], [22] after eliminating high-frequency noise[3], Fig. 1.

If we consider the portion of the $\frac{d P}{d \varphi}$ diagram from the maximum compression rate (point $P^{\prime} m$ ) to the start of combustion (the coordinate of point Pc'), we can see that it can be modeled by the straight line LL' or the portion of the sinusoid SS'. Despite the fact that the Electronic Indicator Lemag documentation "Premet XL, C" [18] says that it is tangent to the inclined section of the $P$ ' chart, by all indications of further actions there is meant a linear approximation of this section [4] . Without taking into account the thermodynamic displacement of the pressure diagram (it is negligibly small), the approximating straight line should cross " 0 " in the TDC position. This position follows from the fact that the rate of pressure change in TDC in this case is zero.

The equation of the straight line $L L^{\prime}$ ' can be written as

$$
L L^{\prime}=b_{0}+b_{1} \varphi,
$$

The TDC coordinate is determined from the condition

$$
\left[L L^{\prime}\right]_{\varphi}^{\prime}=b_{1}=0 .
$$




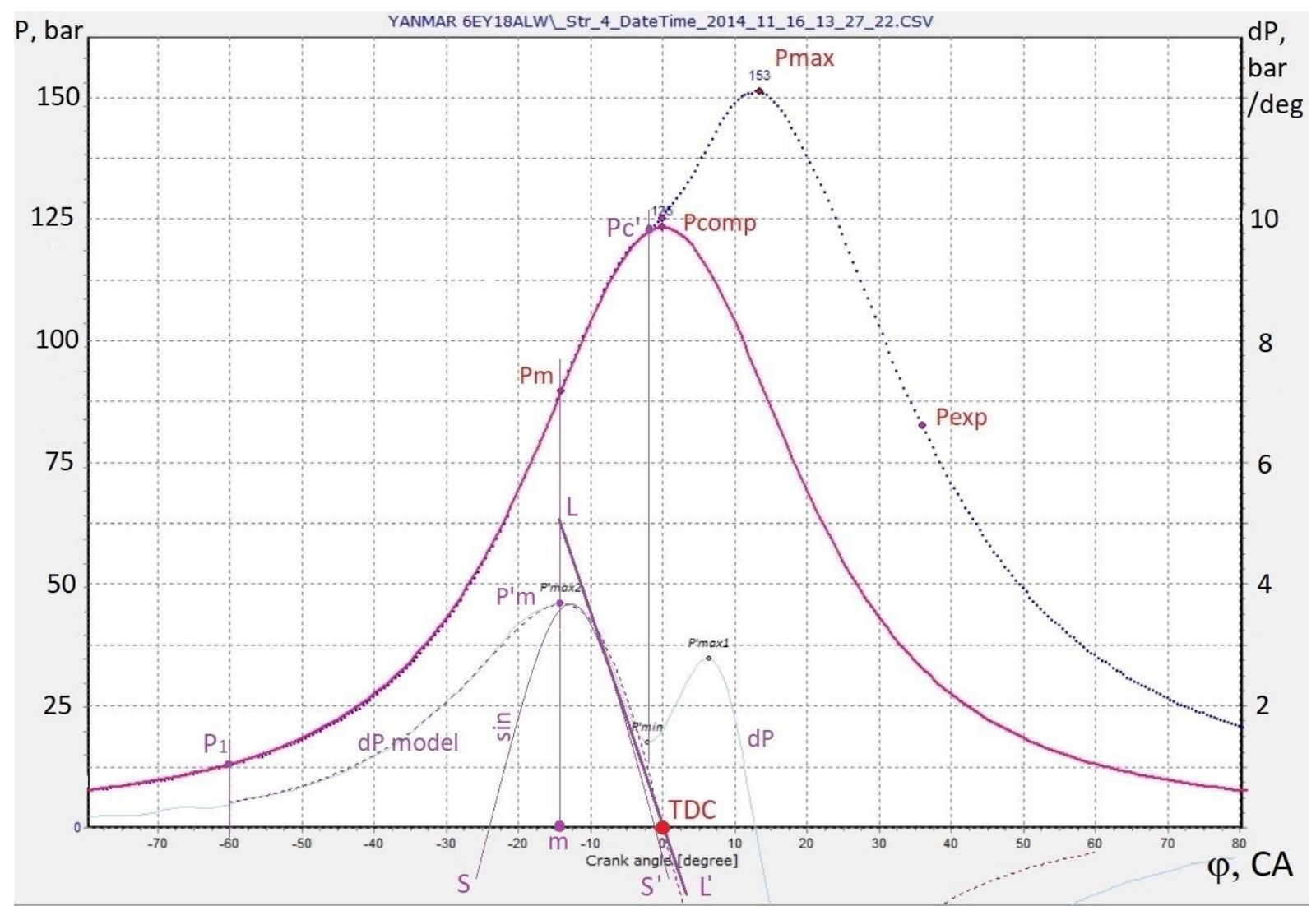

Figure 1. Diagrams $P(\varphi, d e g), d P / d \varphi$ of a medium-speed marine diesel Yanmar 6EY18ALW

A significant problem of the linear regression method, which significantly increases the TDC determination error, appears when the [Pm, Pc'] section of the $\frac{d P}{d \varphi}$ diagram is too small. In Fig. 2 shows the indicator diagram of the MAN 9L58/64 marine engine with a large angle of fuel injection timing.

In the case of early angle of fuel injection timing the $\left[\mathrm{Pm}, \mathrm{Pc}^{\prime}\right]$ section of the $\frac{d P}{d \varphi}$ diagram is shortened, as shown in Fig. 2. In some cases, when the geometrical angles of fuel injection timing reach $15-20^{\circ} \mathrm{CA}$ to $\mathrm{TDC}$, this section disappears altogether. In such cases, the TDC refinement method proposed in the Electronic Indicator Lemag "Premet XL, C" systems will work with a large error [2].

The most stable operation of the TDC determination method using linear regression will be on modern two-stroke marine engines with late fuel injection and the beginning of combustion later than TDC, Fig. 3.

In this case, the [Pm, $\left.\mathrm{Pc}^{\prime}\right]$ section of the $\frac{d P}{d \varphi}$ diagram has a sufficient number of points to build a linear regression model. Moreover, the $\frac{d P}{d \varphi}$ diagram intersects " 0 ", and there is no need to extrapolate the linear model, thereby increasing the probability of error. The TDC position in this case is located inside the [Pm, Pc'] section, which makes it possible to determine the TDC with high accuracy, as, for example, for the Wartsila 6RT-FLEX82C engine (see Fig. 3) [2]. 


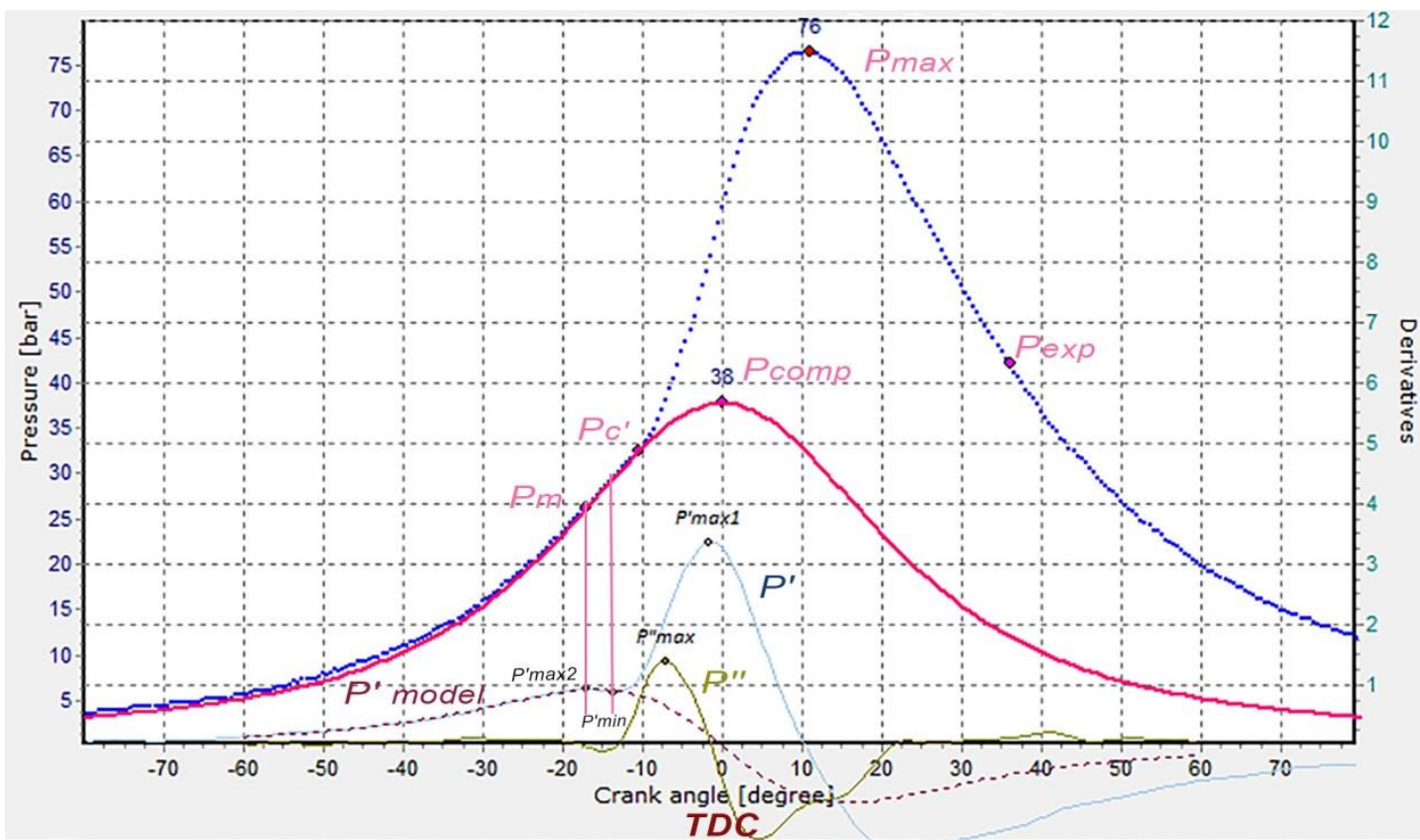

Figure 2. Indicator diagram MAN 9L58/64 with a large angle of fuel injection timing

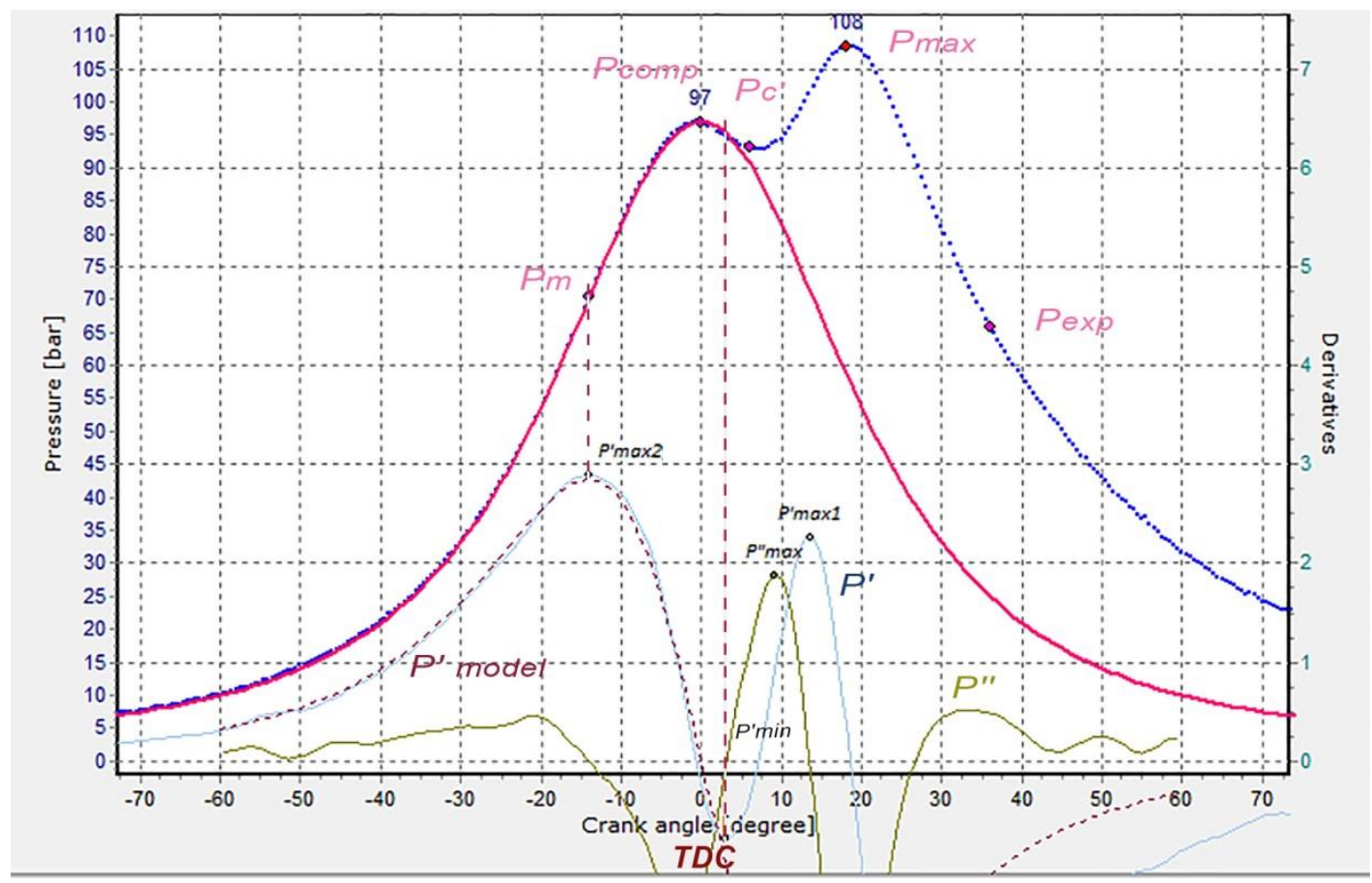

Figure 3. Indicator diagram 6RT-FLEX82C with late injection timing

4.2 Sinusoidal Model. In all cases, the derivatives of the initial diagram $P(t)$ are determined by the method of numerical differentiation, while the high-frequency noise is removed (as shown in ). This makes it possible to further analyze the obtained diagrams $P^{\prime}(t)$ and $P^{\prime \prime}(t)$. Using this analysis, it is possible to determine the coordinates of the extremes $P^{\prime}(t)$ and $P^{\prime \prime}(t)$ in the compression sections and, thus, determine the phase of the combustion beginning Pc' with sufficient accuracy [2]. The $P c^{\prime}$ ' phase 
is approximately the end of the compression process in the cylinder and is the right boundary of the site simulation $\left[P m, P c^{\prime}\right]$.

Obviously, the linear regression model does not accurately describe the nature of the change in pressure in the [Pm, $\left.\mathrm{Pc}^{\prime}\right]$ section, which is not linear in its physical essence. We can even say that with a very large approximation this section is similar to a linear dependence. In addition, in the situations shown in Fig. 2, the TDC correction method proposed in the Electronic Indicator Lemag "Premet XL, C" [18] systems will not work at all or will work poorly.

Obviously, the $\left[\mathrm{Pm}, \mathrm{Pc}^{\prime}\right]$ section of the $\frac{d P}{d \varphi}$ diagram can be modeled with a significantly smaller error by the sinusoidal SS' model (see Fig. 1). In this case

$$
S S^{\prime}=P_{m}^{\prime} \sin (B \varphi+A),
$$

where $B, A$ are the coefficients of the sinusoidal model; $P^{\prime} m$ is the maximum of the first derivative pressure diagram in the compression section [19].

Unlike the linear model, a part of the $\frac{d P}{d \varphi}$ curve to the $P^{\prime} m$ point is used to construct it. When building a sinusoidal model, up to $\vartheta=6 \div 10$ points can be used before the $P m$ coordinate and after the Pc' coordinate (when recording indicator diagrams with a step of 2 points per $1{ }^{\circ} \mathrm{CA}$ ).

4.3 Model equal to zero the first derivative of the pressure under compression. If we use the model of the rate of change in pressure in the compression section obtained from the equation of the polytrophic compression $P V^{n}=$ const, then we can use a large base of experimental points [21]. For example, we can use all the points from the beginning of the compression process (after closing the intake valves) to the start of combustion in the cylinder. However, when analyzing real indicator diagrams, the influence of noise in the areas after the valves are closed is too large, which introduces an additional error in the construction of the regression model. The influence of noise is much less if we consider the portion of the diagram from the coordinate $P_{l} \sim 0.1 P \max$ (see Fig. 1) to the coordinate $P^{\prime} c$ [2]. Thus, the basis for constructing the model $P^{\prime}=0$ is the part of the diagram $\left[\varphi_{i} P_{l}, \varphi_{i} P^{\prime} c-\vartheta\right]$.

The gas pressure in the specified compression section is

$$
\tilde{p}_{\text {comp }}=P_{a}\left(\frac{V_{a}}{V_{\varphi}}\right)^{n 1}
$$

where $P_{a}$ is the pressure at the beginning of compression [5];

$V \varphi$ is the current volume of the cylinder.

$V_{\varphi}=V_{C}+0,5 V_{S}\left[1+\frac{1}{\lambda_{U I}}-\cos \varphi-\frac{1}{\lambda_{U I}} \sqrt{1-\left(\lambda_{U} \sin \varphi\right)^{2}}\right]$

$V_{S}=V_{c}(\varepsilon-1)-$ the volume described by the full stroke of the piston;

$V_{c}$ is the volume of the compression chamber;

$\lambda_{U}=R_{K P} / L_{U I}=S / 2 L_{U I}$ - the ratio of the radius of the crank to the length of the connecting rod.

In relation to the task of TDC determining, we take the value $n l$ constant. According to the recommendations for low-speed and medium-speed engines, the value $n l$ is assumed to be $1.34 \div 1.37$ [21]. Further analysis showed that in the problem of determining the TDC, such an assumption is appropriate and an approximate average value of $n l$ within the indicated limits does not impair the quality of the simulation $d P / d \varphi$. Modeling quality greatly depends on the level of noise on the compression line and on the accuracy of determining the coordinates of the end compression $P c^{\prime}$, modeling right border area [24].

The expression for the rate of pressure change at the compression section can be written

$$
\frac{d P_{\text {comp }}}{d \varphi}=-P_{a} V_{a}^{n 1} n_{1} \frac{1}{V_{\varphi}^{n 1+1}} \cdot \frac{d V_{\varphi}}{d \varphi},
$$

To remove noise in these areas, the authors use the Butterworth digital filter [23], which has a smooth characteristic in the entire frequency range and does not shift the phase of the original signal. 
The results of modeling the rate of pressure change $d P \operatorname{comp} / d \varphi$ in the compression areas before the start of $P^{\prime} c$ combustion on different types of indicator diagrams are shown in Fig. 1-3.

Using a relatively large base of experimental points $\left[\varphi_{i} P_{l}, \varphi_{i} P^{\prime} c-\vartheta\right]$ to build the $d P c o m p / d \varphi$ model gives good results. The model constructed in this way describes with high accuracy the compression process for different types of engines, even in the case of significantly earlier angles of the beginning of fuel combustion in the cylinder, as in Fig. 2.

\section{The Results}

Due to the fact that indicator diagrams are recorded in the computer's memory in the form of time series $P(t)$, the linear regression method is the first method for the analytical determination of TDC. For the linear regression method, the preliminary assessment of the angles of the ${ }^{\circ} \mathrm{CA}$ is not needed.

After the initial determination of the TDC using the linear regression method, a preliminary transformation $f(t) \stackrel{T D C}{\longrightarrow} f(\varphi)$ of several consecutive operating cycles is performed. As a result, the method of sinusoidal approximation of the pressure change rate $d P \operatorname{comp} / d \varphi$ can be applied, which refines the position of the TDC of several cycles.

The final position of the TDC and the translation of the diagrams from the time series to $\varphi$ diagrams $f(t) \stackrel{T D C}{\longrightarrow} f(\varphi)$ is carried out by solving the $P^{\prime}=0$ equation. The initial TDC approximation of several cycles was obtained at the previous step using a sinusoidal model.

The specified algorithm allows you to calculate the position of the TDC with a maximum absolute error of not more than $0.1 \ldots 0.3{ }^{\circ} \mathrm{CA}$. This ensures the required accuracy of the final calculation of the mean indicated pressure with the subsequent calculation of the indicator and effective engine power with a maximum relative error of no more than $2.5 \%$ [4].

Such accuracy in determining the engine power allows predicting its load, diagnosing, calculating specific parameters and energy efficiency coefficients in accordance with IMO requirements [7]. This generally improves the efficiency of marine engines.

The proposed method of analytical determination of TDC and subsequent data synchronization gives the following advantages for portable monitoring working process systems of diesel engines:

- reduces the number of sensors and cables in the indication, which decreases the probability of failures and errors;

- displacements of TDC due to various factors during operation are automatically recognized;

- the impact of the indicator tap channel is automatically recognized [3];

- the error of the method of analytical determination of the TDC and the subsequent synchronization of the indicator diagrams is much smaller $\left(0.1 \ldots 0.3^{\circ}\right.$ crank-shaft angle) than in the hardware method where the error exceeds $0.5^{\circ}$ crank-shaft angle.

\section{Conclusions}

The TDC determination using sensors, used in stationary monitoring systems, requires additional correction due to the displacement of the TDC position mark during engine operation under load. In this case, it is necessary to take into account the change in micro-backlash in the bearings of the crank mechanism and the thermodynamic dis-placement of the maximum compression pressure in the cylinder [25], [26]. For stationary systems, the proposed analytical method can clarify the TDC position determined using sensors [23].

In portable diagnostic systems, the TDC determination is best done immediately by the analytical method. First of all, there are inconveniences during the diagnosis of engines, because additional pickup sensors and their cables must be used. Before installing the sensors, it is necessary to take the engine out of operation. The installed sensors must be calibrated, which is associated with a significant investment of time [22]. All portable systems for parametric engine diagnostics measure the pressure in the working cylinder through the channel of the indicator valve. In this regard, in portable systems, in addition to the aforementioned errors, errors occur such as throttling and delay of the signal, which leads to an additional shift of the TDC position [3]. Thus, recently, most modern portable systems for 
diagnosing marine diesel engines do not use pickup sensors, but use different variants of the algorithmic determination of TDC [29], [30].

\section{References}

[1] Heywood, John B. 1988. Internal combustion engine fundamentals. New York: McGrawHill.

[2] Varbanets R. A., Zalozh V. I., Shakhov A. V., Savelieva I. V., Piterska V. M. Determination of top dead centre location based on the marine diesel engine indicator diagram analysis. Diagnostyka. 2020;21(1):51-60. https://doi.org/10.29354/diag/116585. Available from: http://www.diagnostyka.net.pl/pdf-11658545831?filename=Determination\%20of\%20top\%20dead.pdf

[3] Neumann S, Varbanets R, Kyrylash O, Yeryganov OV, Maulevych VO. Marine diesels working cycle monitoring on the base of IMES GmbH pressure sensors data. Diagnostyka. 2019;20(2):19-26. https://doi:10.29354/diag/104516.

[4] Zalozh, V.I. Efficiency control improvement of diesel engines conditions by using the method of analytical synchronization of monitored data. Dissertation of the Candidate of Technical Sciences. Kyiv, 2020. 162 p. Available from: https://files.duit.edu.ua/uploads/\%D0\%A1\%D0\%B0\%D0\%B9\%D1\%82/3 \%D0\%9D\%D0\% 90\%D0\%A3\%D0\%9A\%D0\%90/\%D0\%A1\%D0\%9F\%D0\%95\%D0\%A6 \%D0\%A0\%D0\% 90\%D0\%94\%D0\%98/\%D0\%94-26-110-01/zalozh_v_i/dis_zalozh.pdf

[5] Varbanets R. Analyse of marine diesel engine performance / R. Varbanets, A. Karianskiy // Journal of Polish CIMAC. Energetic Aspects. - Gdansk: Faculty of Ocean Engineering and Ship Technology Gdansk University of Technology, 2012. - Vol. 7, No. 1. - C. 269-275. https://doi.org/10.33082/td.2018.2-3.09

[6] Varbanets R., Zalozh V. The analytical synchronization of the monitoring data of the working process in transport diesel engines operating. Internet Conference. Kyiv, 2019. Available from:

http://old.duit.edu.ua/wpcontent/uploads/2019/12/\%D0\%9C\%D0\%90\%D0\%A2\%D0\%95\%D0\%A0\%D0\%86\%D0\% 90\%D0\%9B\%D0\%98-

\%D0\%92\%D0\%A1\%D0\%95\%D0\%A3\%D0\%9A\%D0\%A0\%D0\%90\%D0\%87\%D0\%9D \% D0\%A1\%D0\%AC\%D0\%9A\%D0\%9E\%D0\%87\%D0\%98\%D0\%9D\%D0\%A2\%D0\%95\%D0\%A0\%D0\%9D\%D0\%95\%D0\%A2\%D0\%9A \%D0\%9E\%D0\%9D\%D0\%A4\%D0\%95\%D0\%A0\%D0\%95\%D0\%9D\%D0\%A6\% D0\%86\%D0\%87 \%D0\%9A\%D0\%98\%D0\%87\%D0\%92 \%D0\%94\%D0\%A3\%D0\%86\%D 0\%A2 2019.pdf

[7] Resolution MEPC.282(70). 2016 Guidelines for the development of a ship energy efficiency management plan (SEEMP) [Internet]. International Maritime Organization (IMO). Available from: https://qi.decoplagerent.com/230.html

[8] IMES cylinder pressure sensors. Available from: https://www.imes.de

[9] Pressure and TDC sensors from Kistler. Available from: https://www.kistler.com

[10] AVL OT-sensor 428. Available from: https://www.avl.com

[11] Ding Y, Stapersma D, Grimmelius HT. Cylinder process simulation with heat release analysis in diesel engine. Conference: Power and Energy Engineering Conference, 2009. APPEEC 2009. Asia-Pacific. https://doi.org/10.1109/APPEEC.2009.4918248

[12] Tazerout, M., Le Corre, O., Rousseau, S., TDC Determination in IC Engines Based on the Thermodynamic Analysis of the Temperature-Entropy Diagram, SAE Technical Paper 199901-1489, 1999. https://doi.org/10.4271/1999-01-1489

[13] Staś M. "An Universally Applicable Thermodynamic Method for T.D.C. Determination," SAE Technical Paper 2000-01-0561, 2000. DOI: https://doi.org/10.4271/2000-01-0561

[14] Polanowski, S., Determination of location of Top Dead Centre and compression ratio value 
on the basis of ship engine indicator diagram. Polish Maritime Research, No. 2(56), Vol. 15, 2008. https://doi.org/10.2478/v10012-007-0065-2

[15] Per Tunest ${ }^{\circ}$ al, Model Based TDC Offset Estimation from Motored Cylinder Pressure Data, Proceedings of the 2009 IFAC Workshop on Engine and Powertrain Control, Simulation and Modeling IFP, Rueil-Malmaison, France, Nov 30 - Dec 2, 2009, https://doi.org/10.3182/20091130-3-FR-4008.00032

[16] Nilsson, Y. and Eriksson, L., Determining TDC Position Using Symmetry and Other Methods, SAE Technical Paper 2004-01-1458, 2004, https://doi.org/10.4271/2004-01-1458.

[17] Emiliano Pipitone, Alberto Beccari. Determination Of Tdc In Internal Combustion Engines By A Newly Developed Thermodynamic Approach. Applied Thermal Engineering, Elsevier, 2009, https://doi.org/10.1016/ j.applthermaleng.2010.04.012

[18] Lemag PreMet XL, C Cylinder Pressure Indicator. Available from: http://www.lemag.de/

[19] M. J. D. Powell, An efficient method for finding the minimum of a function of several variables without calculating derivatives, The Computer Journal, Volume 7, Issue 2, 1964, Pages 155-162, https://doi.org/10.1093/comjnl/7.2.155

[20] Tazerout, M., Le Corre, O., Stouffs, P. , Compression Ratio and TDC calibrations using Temperature - Entropy Diagram, SAE Technical Paper 1999-01-3509, 1999. https://doi.org/10.4271/1999-01-3509

[21] Yeryganov, O., and Varbanets, R. (2018). Features of the fastest pressure growth point during compression stroke. Diagnostyka, 19(2), pp.71-76. https://doi.org/10.29354/diag/89729

[22] Neumann S. High temperature pressure sensor based on thin film strain gauges on stainless steel for continuous cylinder pressure control. CIMAC Congress 2001, Hamburg. Digest; p. $1-12$.

[23] Varbanets, R. A. Diagnostic control of the working process of marine diesel engines in operation. Dissertation of the Doctor of Technical Sciences. Odessa, 2010. 314 p.

[24] Varbanets, R., Karianskyi, S., Rudenko, S., Gritsuk, I. et al., "Improvement of Diagnosing Methods of the Diesel Engine Functioning under Operating Conditions," SAE Technical Paper 2017-01-2218, 2017.

[25] Žvar Baškovič, U.; Vihar, R.; Mele, I.; Katrašnik, T. A New Method for Simultaneous Determination of the TDC Offset and the Pressure Offset in Fired Cylinders of an Internal Combustion Engine. Energies 2017, 10(1), 143; https://doi.org/10.3390/en10010143.

https://www.mdpi.com/1996-1073/10/1/143

[26] Pawletko, Rafał. (2014). Increasing the accuracy of the mean indicated pressure determination by appointment of the tdc cylinder position. Journal of kones. Powertrain and Transport. 21. 397-404. DOI: 10.5604/12314005.1130494.

[27] Ship Energy Efficiency Management [Internet]. Available from: https://docplayer.net/24904791-Ship-energy-efficiency-management.html

[28] International Maritime Organization (IMO) [Internet]. Available from: http://www.imo.org/en/KnowledgeCentre/IndexofIMOResolutions/Marine-Environment-

Protection-Committee-\%28MEPC\%29/Pages/default.aspx

[29] KOWALAK P. Marine engine indicated power uncertainty determination. Combustion Engines. 2015;161(2):3-13. Available from: http://www.combustion-engines.eu/pdf116885-46099?filename $=$ Marine $\% 20$ engine $\% 20$ indicated.pdf

[30] Toru Miyamoto, Hirokazu Hasegawa, Masato Mikami, Naoya Kojima, Hajime Kabashima, Yasuhiro Urata, Effect of hydrogen addition to intake gas on combustion and exhaust emission characteristics of a diesel engine, International Journal of Hydrogen Energy, Volume 36, Issue 20, 2011, Pages 13138-13149, ISSN 0360-3199, https://doi.org/10.1016/j.ijhydene.2011.06.144. 